\title{
The blue to red supergiant ratio in young clusters at various metallicities
}

\author{
P. Eggenberger, G. Meynet, and A. Maeder
}

\author{
Geneva Observatory CH-1290 Sauverny, Switzerland \\ e-mail: Georges.Meynet@obs.unige.ch, Andre.Maeder@obs.unige.ch
}

Received 6 December 2001 / Accepted 18 February 2002

\begin{abstract}
We present new determinations of the blue to red supergiant ratio $(B / R)$ in young open clusters at various metallicities. For this purpose, we examine the HR diagrams of 45 clusters in the Galaxy and of 4 clusters in the Magellanic Clouds. The identification of supergiants is based on spectroscopic measurements (with photometric counts to check the results). The new counts confirm the increase of the $B / R$ ratio when the metallicity increases with the following normalized relation: $\frac{B / R}{(B / R)_{\odot}} \cong 0.05 \cdot \mathrm{e}^{3 \frac{Z}{Z} \odot}$, where $Z_{\odot}=0.02$ and $(B / R) \odot$ is the value of $B / R$ at $Z_{\odot}$ which depends on the definition of $B$ and $R$ and on the age interval considered (e.g. for spectroscopic counts including clusters with $\log$ age between 6.8 and $7.5,(B / R)_{\odot} \cong 3$ when $B$ includes $\mathrm{O}, \mathrm{B}$ and A supergiants).
\end{abstract}

Key words. stars: evolution - stars: supergiants - galaxies: Magellanic Clouds

\section{Introduction}

The variation with metallicity $Z$ of the number of blue and red supergiants is important in relation to the nature of the supernova progenitors in different environments (Langer 1991a,b) and its effects on the luminous star populations in galaxies (e.g. Cervino \& Mas-Hesse 1994; Origlia et al. 1999). This ratio also constitutes an important and sensitive test for stellar evolution models, because it is very sensitive to mass loss, convection and mixing processes (Langer \& Maeder 1995). Stellar evolution models can usually, by adjustement of parameters such as the rate of mass loss by stellar winds, reproduce the observed ratio for a given metallicity, but are not able to reproduce its variation with the metallicity. Thus, the problem of the blue to red supergiant ratio ( $B / R$ ratio) remains one of the most severe problems in stellar evolution.

Since the last studies of the $B / R$ ratio in the Galaxy and in the Magellanic Clouds more than fifteen years ago (Meylan \& Maeder 1982; Humphreys \& McElroy 1984), many new spectroscopic and photometric measurements have been performed. Our aim here is to reexamine this question accounting for these recent observational improvements. We chose to use stellar clusters instead of field stars for obvious reasons: stars are at the same distance, with the same age and above all have the same chemical

Send offprint requests to: P. Eggenberger,

e-mail: eggenbe7@etu.unige.ch composition. Moreover, the knowledge of the age of the clusters enables us to estimate the initial masses of the supergiants used to derive the $B / R$ ratio.

Thus, the present work is in the continuity of the work of Meylan \& Maeder (1982). However, the present study presents two significant improvements. First, the identification of supergiants is based on spectroscopic measurements instead of on photometric colours. Spectroscopic measurements allow a more accurate differentiation between supergiants and main sequence stars (see however the discussion in Sect. 4). Second, the number of stellar clusters in our galactic sample is increased by a factor of four.

In Sect. 2, we review the various studies about the $B / R$ ratio. Section 3 presents the new counts. Our results are discussed in Sect. 4, and conclusions are given in Sect. 5.

\section{Previous studies}

Walker (1964) observed that the $B / R$ ratio in M 33 decreases when the distance to the centre of the galaxy increases. Van den Bergh (1968) explained this variation by a radial metallicity gradient in the disk of M 33, the metallicity being higher in the inner parts. Humphreys \& Sandage (1980) reexamined the $B / R$ ratio in $M 33$ and confirmed its decrease when the galactocentric distance increases. Freedman (1985) contested the results of Humphreys \& Sandage (1980) concerning the variation of the $B / R$ ratio in $\mathrm{M} 33$ and concluded that this was 
just an effect of incompleteness. However, Ivanov (1998) concluded that the diminution was real and was in good agreement with the radial metallicity gradient observed in M33 (McCarthy et al. 1995).

Hartwick (1970) noticed the decrease of the $B / R$ ratio in the Milky Way as the galactocentric radius increases. Robertson (1973, 1974), Hagen \& van den Bergh (1974) compared theoretical evolutionary tracks to observed HR diagrams (HRD) of stellar clusters in the Galaxy and in the Large Magellanic Cloud (LMC). They attributed the observed differences between the HRD to differences in the metal content.

Humphreys (1979a) investigated the variation of the $B / R$ ratio in the LMC. She concluded that the radial variation of this ratio must be weak or absent, in agreement with the study of the chemical composition of the HII regions made by Pagel et al. (1978). In a study of the supergiants in the Galaxy and in the LMC, Humphreys \& Davidson (1979) found a decrease of the $B / R$ ratio when the metallicity decreases. Cowley et al. (1979) examined the variation of the $B / R$ ratio across the face of the LMC and found that it increases by a factor of 1.8 when the metallicity increases by a factor of 1.2.

The first study of the $B / R$ ratio using stellar clusters in the Galaxy, the LMC and the SMC (Small Magellanic Cloud) was made by Meylan \& Maeder (1982), who concluded to a steep increase of $B / R$ when the metallicity increases, with a difference of about an order of magnitude in $B / R$ between the Galaxy and the SMC. Humphreys \& McElroy (1984) reexamined the $B / R$ ratio in the Galaxy, the LMC and the SMC, and gave new values which accounted for the incompleteness of the data. They found a $B / R$ ratio about ten times higher in the central parts of the Galaxy than in the SMC.

Langer \& Maeder (1995) summarized some results about the values of the $B / R$ ratio and gave a $B / R$ ratio approximately six times larger in the solar neighborhood than in the SMC. After comparison of different stellar models with observations, they concluded that most massive star models have problems reproducing the decrease of $B / R$ when the metallicity decreases. Deng et al. (1996) used the supergiant stars catalogue from Blaha \& Humphreys (1989) to make new counts in the Galaxy and in the LMC. They found a $B / R$ ratio about five times higher in the Galaxy than in the LMC.

To summarize the $B / R$ ratio appears to be an increasing function of metallicity. This trend is observed by studies in the Galaxy, the Magellanic Clouds as well as in the Triangulum galaxy. In this work we shall take the opportunity of recent improvements brought to our knowledge of Galactic and Magellanic clusters to reexamine carefully this question.

\section{Results from new cluster studies in the Galaxy, LMC and SMC}

\subsection{Spectroscopic counts}

We select in the Webda database (Mermilliod 1995; http://obswww.unige.ch/webda) young clusters with $\log$ age between 6.8 and 7.5, corresponding to masses at the turn off between $8 M_{\odot}$ and $24 M_{\odot}$ and to initial masses of the supergiants between about 8 and $30 M_{\odot}$ (Meynet et al. 1993). Let us note that this interval of masses for the supergiants probes a region of the HR diagram below the observed upper limit for the luminosity of the red supergiants, i.e. below $M_{\text {bol }} \sim-9.5$ (Humphreys \& Davidson 1979, 1984).

We only keep the clusters with spectroscopic measurements for the brightest stars in order to distinguish supergiants from main sequence stars. In this way, 45 clusters are selected. For each cluster, we count the number of supergiant stars of spectral type O, B, A, K and M. In a few cases, the spectroscopic class for the same star is different depending on the authors. In these cases, we adopt the most recent determination. The galactocentric distance $\mathrm{R}$ of each cluster is calculated from the values of its galactic coordinates and from its distance to the sun given by the Webda database. For each galactocentric radius, a metallicity is associated by taking a value of $Z=0.02$ at the solar position (we take $\mathrm{R}_{\odot}=8.5 \mathrm{kpc}$ for the solar galactocentric distance) and an averaged radial metallicity gradient of $-0.08 \mathrm{dex} \mathrm{kpc}^{-1}$. This value has been chosen from various studies of the galactic gradient determined by means of spectroscopic and photometric indices of stellar populations in open clusters (see the discussion by Alibés et al. 2001).

The results are given in Table 1 , where $B$ represents the number of $\mathrm{O}, \mathrm{B}$ and $\mathrm{A}$ supergiants, while $R$ is the number of $\mathrm{K}$ and $\mathrm{M}$ supergiants. $N_{\mathrm{O}}, N_{\mathrm{B}}, N_{\mathrm{A}}, N_{\mathrm{K}}$ and $N_{\mathrm{M}}$ indicate the number of supergiants with spectral type $\mathrm{O}, \mathrm{B}, \mathrm{A}, \mathrm{K}$ and $\mathrm{M}$ respectively. Each cluster age is given in logarithm and taken from the Webda database. The region noted GC refers to the region inside the solar circle and includes all the clusters with a galactocentric distance shorter than $8.5 \mathrm{kpc}$. Likewise, the GAC region corresponds to the region outside the solar circle and includes the clusters with a galactocentric distance higher than $8.5 \mathrm{kpc}$.

On the basis of these results, we examined the variation of the $B / R$ ratio with the galactocentric radius by grouping open clusters in different distance bins. In Fig. 1, two different binnings are shown. In the first one, clusters are grouped in three main galactocentric distance intervals $(6.5-8.0,8.0-9.0$ and $9.0-11.5 \mathrm{kpc})$, in order to have the same number of clusters (15) in each bin. We also separate the clusters in five distance intervals $(6.5-7.5,7.5-$ $8.0,8.0-8.5,8.5-10.0$ and $10.0-11.5 \mathrm{kpc})$ to check whether the variation of the $B / R$ ratio observed when considering three intervals is still present (due to the small number of supergiants counted for each clusters, it is not pertinent 
Table 1. Number of supergiant stars in galactic open clusters with $\log$ age between 6.8 and 7.5 . The quantity $\mathrm{R}$ is the galactocentric radius and $Z$ is the metallicity. GC means clusters toward the galactic centre, GAC toward the galactic anticentre. The identification of supergiants is based on spectroscopic measurements. $B$ includes $\mathrm{O}$, B, A supergiants and $R$ includes $\mathrm{K}$, $\mathrm{M}$ supergiants (see text).

\begin{tabular}{|c|c|c|c|c|c|c|c|c|c|c|}
\hline Cluster & $\overline{~ l o g}$ age [y] & $\overline{\mathrm{R}[\mathrm{kpc}]}$ & $\overline{Z Z}$ & $\bar{B}$ & $\bar{R}$ & $\overline{N_{\mathrm{O}}}$ & $\overline{N_{\mathrm{B}}}$ & $\overline{N_{\mathrm{A}}}$ & $N_{\mathrm{K}}$ & $\overline{N_{\mathrm{M}}}$ \\
\hline \multicolumn{11}{|l|}{$\mathrm{GC}$} \\
\hline NGC 6611 & 6.88 & 6.85 & 0.027 & 2 & 0 & 0 & 2 & 0 & 0 & 0 \\
\hline NGC 6604 & 6.81 & 6.91 & 0.027 & 2 & 0 & 1 & 1 & 0 & 0 & 0 \\
\hline Pismis 20 & 6.86 & 7.06 & 0.026 & 4 & 0 & 0 & 4 & 0 & 0 & 0 \\
\hline NGC 6530 & 6.87 & 7.18 & 0.025 & 1 & 0 & 0 & 1 & 0 & 0 & 0 \\
\hline NGC 6613 & 7.22 & 7.25 & 0.025 & 3 & 0 & 0 & 3 & 0 & 0 & 0 \\
\hline Trumpler 27 & 7.06 & 7.29 & 0.025 & 8 & 1 & 1 & 7 & 0 & 0 & 1 \\
\hline NGC 6231 & 6.84 & 7.32 & 0.025 & 3 & 0 & 2 & 1 & 0 & 0 & 0 \\
\hline NGC 6664 & 7.16 & 7.45 & 0.024 & 0 & 1 & 0 & 0 & 0 & 1 & 0 \\
\hline NGC 4755 & 7.22 & 7.60 & 0.024 & 5 & 1 & 0 & 4 & 1 & 0 & 1 \\
\hline NGC 6514 & 7.37 & 7.69 & 0.023 & 3 & 0 & 0 & 1 & 2 & 0 & 0 \\
\hline NGC 6823 & 6.82 & 7.71 & 0.023 & 1 & 0 & 0 & 1 & 0 & 0 & 0 \\
\hline NGC 5281 & 7.15 & 7.85 & 0.023 & 1 & 0 & 0 & 0 & 1 & 0 & 0 \\
\hline NGC 3603 & 6.84 & 7.92 & 0.022 & 3 & 0 & 2 & 1 & 0 & 0 & 0 \\
\hline IC 2944 & 6.82 & 7.92 & 0.022 & 3 & 1 & 1 & 2 & 0 & 0 & 1 \\
\hline NGC 3766 & 7.16 & 7.95 & 0.022 & 2 & 2 & 0 & 2 & 0 & 2 & 0 \\
\hline NGC 3590 & 7.23 & 8.05 & 0.022 & 1 & 0 & 0 & 1 & 0 & 0 & 0 \\
\hline Trumpler 18 & 7.19 & 8.11 & 0.021 & 1 & 0 & 0 & 1 & 0 & 0 & 0 \\
\hline Collinder 228 & 6.83 & 8.11 & 0.021 & 2 & 1 & 1 & 1 & 0 & 0 & 1 \\
\hline Trumpler 15 & 6.93 & 8.14 & 0.021 & 1 & 1 & 1 & 0 & 0 & 0 & 1 \\
\hline Bochum 10 & 6.86 & 8.14 & 0.021 & 1 & 0 & 0 & 1 & 0 & 0 & 0 \\
\hline NGC 6871 & 6.96 & 8.17 & 0.021 & 2 & 0 & 0 & 2 & 0 & 0 & 0 \\
\hline NGC 3293 & 7.01 & 8.18 & 0.021 & 2 & 1 & 1 & 1 & 0 & 0 & 1 \\
\hline IC 2581 & 7.14 & 8.23 & 0.021 & 2 & 0 & 0 & 1 & 1 & 0 & 0 \\
\hline NGC 6913 & 7.11 & 8.32 & 0.021 & 3 & 0 & 1 & 2 & 0 & 0 & 0 \\
\hline NGC 6910 & 7.13 & 8.35 & 0.021 & 1 & 0 & 0 & 1 & 0 & 0 & 0 \\
\hline Berkeley 87 & 7.15 & 8.37 & 0.020 & 0 & 1 & 0 & 0 & 0 & 0 & 1 \\
\hline \multicolumn{11}{|l|}{ GAC } \\
\hline Collinder 135 & 7.41 & 8.62 & 0.020 & 0 & 1 & 0 & 0 & 0 & 1 & 0 \\
\hline Trumpler 37 & 7.05 & 8.67 & 0.019 & 5 & 2 & 0 & 4 & 1 & 0 & 2 \\
\hline Collinder 121 & 7.05 & 8.77 & 0.019 & 4 & 1 & 0 & 4 & 0 & 1 & 0 \\
\hline NGC 1976 & 7.11 & 8.83 & 0.019 & 0 & 1 & 0 & 0 & 0 & 1 & 0 \\
\hline NGC 7419 & 7.28 & 9.05 & 0.018 & 0 & 5 & 0 & 0 & 0 & 0 & 5 \\
\hline NGC 7235 & 7.07 & 9.53 & 0.017 & 1 & 1 & 0 & 1 & 0 & 1 & 0 \\
\hline NGC 2244 & 6.90 & 9.81 & 0.016 & 0 & 1 & 0 & 0 & 0 & 1 & 0 \\
\hline NGC 2384 & 6.90 & 9.86 & 0.016 & 1 & 0 & 1 & 0 & 0 & 0 & 0 \\
\hline NGC 663 & 7.21 & 9.86 & 0.016 & 7 & 1 & 0 & 7 & 0 & 0 & 1 \\
\hline NGC 957 & 7.04 & 9.89 & 0.015 & 1 & 0 & 0 & 1 & 0 & 0 & 0 \\
\hline NGC 654 & 7.15 & 9.91 & 0.015 & 1 & 0 & 0 & 0 & 1 & 0 & 0 \\
\hline IC 1805 & 6.82 & 9.92 & 0.015 & 1 & 0 & 1 & 0 & 0 & 0 & 0 \\
\hline NGC 581 & 7.34 & 10.00 & 0.015 & 1 & 2 & 0 & 1 & 0 & 0 & 2 \\
\hline NGC 869 & 7.07 & 10.07 & 0.015 & 6 & 1 & 0 & 6 & 0 & 0 & 1 \\
\hline NGC 457 & 7.32 & 10.13 & 0.015 & 1 & 1 & 0 & 0 & 1 & 0 & 1 \\
\hline NGC 884 & 7.03 & 10.29 & 0.014 & 4 & 5 & 0 & 2 & 2 & 0 & 5 \\
\hline NGC 2439 & 7.25 & 10.64 & 0.013 & 1 & 1 & 0 & 1 & 0 & 0 & 1 \\
\hline NGC 2414 & 6.98 & 10.99 & 0.013 & 1 & 0 & 0 & 1 & 0 & 0 & 0 \\
\hline Ruprecht 55 & 6.85 & 11.12 & 0.012 & 1 & 0 & 0 & 1 & 0 & 0 & 0 \\
\hline
\end{tabular}

to adopt a finer binning). The metallicity of each bin is obtained by averaging the metallicities of all the clusters in this bin. We precise here that the metallicities are not obtained from stars in each clusters, but from an adopted metallicity gradient of $-0.08 \mathrm{dex} \mathrm{kpc}^{-1}$. In this way, we assign a metallicity for each distance interval mentioned above (e.g. for the $\mathrm{GC}$ and GAC region we take respectively $Z=0.023$ and $Z=0.016$ ). The increase of the $B / R$ ratio with the metallicity is shown in Fig. 1 and in Table 3.

In order to obtain values of the $B / R$ ratio for lower metallicities, we select young clusters in the Magellanic Clouds. According to a discussion by Maeder et al. (1999), the average value of the metallicity is $Z=0.007$ for the
LMC and $Z=0.002$ for the SMC. Without sufficient spectroscopic data for the young clusters of the LMC, it is not possible to give a reliable value for $B / R$ in the LMC. In the SMC, the only cluster satisfying the age criteria and having spectroscopic measurements is NGC 330 (log age $=7.00$ according to Cassatella et al. 1996). This cluster has been well studied and so different values exist for the $B / R$ ratio: $B / R=7 / 12$ (Cayrel et al. 1988), $B / R=9 / 15$ (Carney et al. 1985; Brocato \& Castellani 1992; Bomans \& Grebel 1994), $B / R=12 / 15$ (Grebel \& Richtler 1992). In these ratios, $B$ only includes B supergiants. Thus, the value of the $B / R$ ratio for NGC 330 lies somewhere between 0.5 and 0.8 (when only B supergiants 


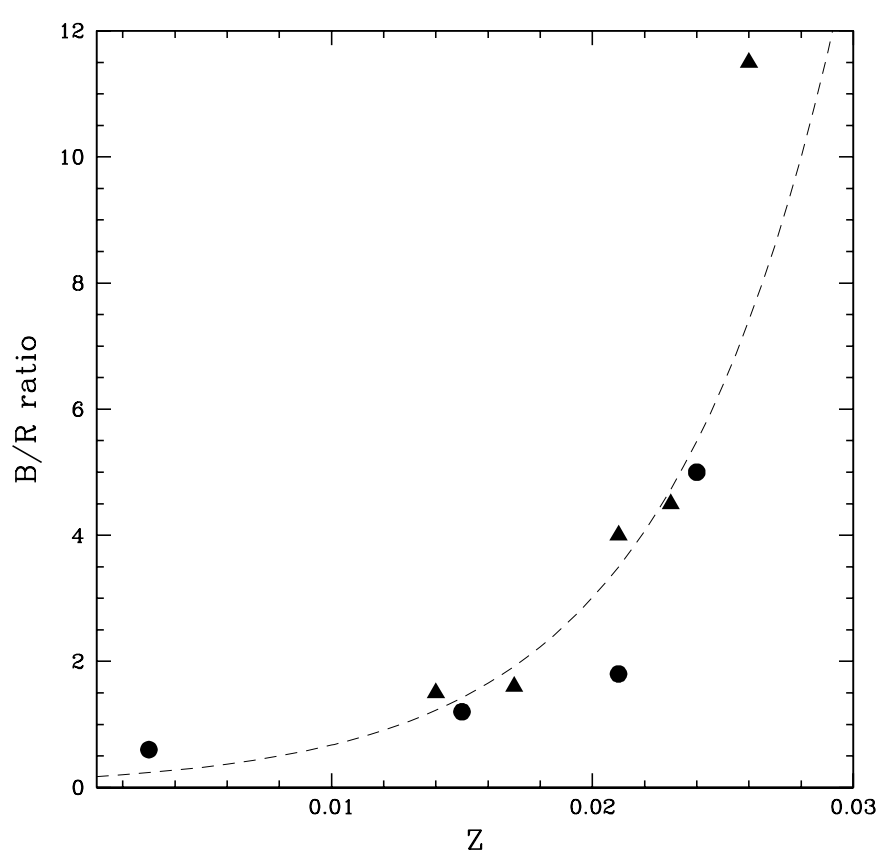

Fig. 1. $B / R$ ratio in the Galaxy and the SMC for clusters with $\log$ age between 6.8 and 7.5. The distinction between blue and red supergiants is based on spectroscopic measurements. The triangles refer to $B$ including $\mathrm{O}, \mathrm{B}$ and $\mathrm{A}$ supergiants and correspond to five different distance intervals in the Galaxy (6.5-7.5, 7.5-8.0, 8.0-8.5, 8.5-10.0 and 10.0-11.5 pc). The dots refer to $B$ including only $\mathrm{B}$ supergiants; they correspond to one value for the SMC and three distance intervals in the Galaxy (6.5-8.0, 8.0-9.0 and 9.0-11.5 kpc). The dashed curve corresponds to the fit for $B$ including $\mathrm{O}, \mathrm{B}$ and $\mathrm{A}$ supergiants with $(B / R)_{\odot}=3.0$ (see text).

are counted). The value of 0.6 is currently accepted and is reported in Fig. 1.

\subsection{Photometric counts}

In order to complete counts based on spectroscopic measurements, we perform counts based on colour indices in the same way as Meylan \& Maeder (1982). To this purpose, we select among the 45 open clusters mentioned in Table 1, those having photometric measurements down to $M_{v}=-2$ and with at least 15 stars brighter than this magnitude. Hence 23 clusters are selected. The distinction between blue and red supergiants is made in the following way: when $(B-V)_{0} \leq 0$, the star is considered to have a $\mathrm{O}, \mathrm{B}$ or $\mathrm{A}$ spectral type and is included in $B$, whereas for $(B-V)_{0} \geq 1.2$, the star is considered to be a $\mathrm{K}$ or a $\mathrm{M}$ and is included in $R$. This distinction remains valid for the SMC, where late-type supergiants are of earlier type than in the Galaxy and LMC (Humphreys 1979b; Elias et al. 1985). We then count all the stars in these two colour intervals down to a certain limit $M_{v l i m}$ of the absolute magnitude. In order to be sure that the results do not depend on the value of this arbitrary chosen limit, the counts are made for four different limits: $M_{v \lim }=-3.25$, $M_{v \lim }=-3.0, M_{v \lim }=-2.5$ and $M_{v \lim }=-2.0$. The
23 selected clusters and the results are listed in Table 2. Because of the small number of clusters selected in this case, it is not pertinent to subdivide the clusters in several distance intervals or age intervals, and so we consider all the clusters with $\log$ age between 6.8 and 7.5 and only the two main regions GC and GAC to calculate the $B / R$ ratio (see Table 3 ).

For the Magellanic Clouds, we use the data from Elson (1991), except for the SMC cluster NGC 330 (Vallenari 1994). Cluster ages are taken from Cassatella et al. (1996). The same criteria of selection as those used for galactic clusters give a total of 3 clusters selected in the LMC. In the SMC, NGC 330 is the only cluster satisfying both the criteria for age and for the number of stars brighter than $M_{v}=-2$. The counts are made in exactly the same way as for the Milky Way. The selected clusters and the results of the counts are given in Table 2, while Table 3 summarizes the values of the $B / R$ ratio found in the four main regions (GC, GAC, LMC and SMC). Let us note here that our selected clusters have a mean age approximately the same in the GC, GAC and SMC regions (respectively 7.0, 7.1 and 7.0) which is a nice feature for the comparisons performed here.

From the results for spectroscopic and photometric counts, we find the following average relation:

$\frac{B / R}{(B / R)_{\odot}} \cong 0.05 \cdot \mathrm{e}^{3 \frac{Z}{z_{\odot}}}$

where $Z_{\odot}=0.02$ and $(B / R)_{\odot}$ is the value of $B / R$ at $Z_{\odot}$ which depends on the definition of $B$ and $R$ and on the age interval considered. For clusters with log age between 6.8 and 7.5 (initial masses of the supergiants between $\sim 8$ and $\left.30 M_{\odot}\right),(B / R)_{\odot} \cong 3.0$ for spectroscopic counts when $B$ includes $\mathrm{O}, \mathrm{B}$ and $\mathrm{A}$ supergiants, and $(B / R)_{\odot} \cong 2.5$ when only $\mathrm{B}$ supergiants are counted. For photometric counts, $(B / R)_{\odot}$ equals $17.2,18.5,23.0$ and 24.0 for $M_{v \lim }=-3.25, M_{v \lim }=-3.0, M_{v \lim }=-2.5$ and $M_{v \lim }=-2.0$, respectively.

\section{Discussion of the results}

Figure 1 clearly shows that the present study, based on the most recent spectroscopic measurements found in the literature, confirms the trend already found in previous works, namely the decrease of the $B / R$ ratio with the metallicity. In the Galaxy, $B / R$ is approximately 3 times higher in the GC than in the GAC (Table 3). This variation can also be observed by separating the Galaxy in three or five galactocentric distance intervals. Moreover, the $B / R$ ratio in the SMC confirms the results obtained for the Galaxy, with a value approximately 2 times lower than in the GAC region. Figure 1 shows also that the decrease is stronger at high metallicity than at low metallicity. This is in good agreement with recent results from Massey (2002) who found a $B / R$ ratio only slightly higher in the LMC than in the SMC.

Concerning photometric counts, we can see in Table 3 that $B / R$ is higher in the GC region than in the GAC 
Table 2. Number counts down to a limit magnitude $M_{v \lim }$. The values of $\left(m_{v}-M_{v}\right)_{0}$ and $E(B-V)$ mentioned for the galactic clusters are from the Webda database (Mermilliod 1995) and from van den Bergh (1998) for the clusters in the Magellanic Clouds. $B$ includes stars with $(B-V)_{0} \leq 0$ and $R$ stars with $(B-V)_{0} \geq 1.2$ (see text).

\begin{tabular}{|c|c|c|c|c|c|c|c|c|c|c|c|c|}
\hline Cluster & \multirow[t]{2}{*}{ age } & $E(B-V)$ & $\left(m_{v}-M_{v}\right)_{0}$ & $Z$ & \multicolumn{2}{|c|}{$M_{v \lim }=-3.25$} & \multicolumn{2}{|c|}{$\bar{M}_{v \lim }=-3.0$} & \multicolumn{2}{|c|}{$M_{v \lim }=-2.5$} & \multicolumn{2}{|c|}{$\begin{array}{c}M_{v \lim }=-2.0 \\
B\end{array}$} \\
\hline GC & & & & & & & & & & & & \\
\hline NGC 6611 & 6.88 & 0.78 & 11.21 & 0.027 & 15 & 0 & 16 & 0 & 21 & 0 & 35 & 0 \\
\hline NGC 6530 & 6.87 & 0.33 & 10.62 & 0.025 & 6 & 0 & 9 & 0 & 13 & 0 & 16 & 0 \\
\hline NGC 6231 & 6.84 & 0.44 & 10.47 & 0.025 & 13 & 0 & 14 & 0 & 16 & 0 & 28 & 0 \\
\hline NGC 4755 & 7.22 & 0.39 & 11.48 & 0.024 & 13 & 1 & 16 & 1 & 28 & 1 & 35 & 1 \\
\hline NGC 6823 & 6.82 & 0.85 & 11.39 & 0.023 & 17 & 0 & 18 & 0 & 30 & 0 & 45 & 0 \\
\hline NGC 3603 & 6.84 & 1.34 & 12.80 & 0.022 & 37 & 2 & 42 & 3 & 63 & 3 & 91 & 6 \\
\hline IC 2944 & 6.82 & 0.32 & 11.27 & 0.022 & 13 & 1 & 16 & 1 & 21 & 1 & 25 & 1 \\
\hline NGC 3766 & 7.16 & 0.18 & 11.21 & 0.022 & 5 & 2 & 11 & 2 & 16 & 2 & 23 & 2 \\
\hline Collinder 228 & 6.83 & 0.34 & 11.71 & 0.021 & 4 & 0 & 7 & 0 & 13 & 0 & 18 & 0 \\
\hline NGC 6871 & 6.96 & 0.44 & 10.99 & 0.021 & 15 & 0 & 16 & 0 & 20 & 1 & 25 & 1 \\
\hline NGC 3293 & 7.01 & 0.26 & 11.83 & 0.021 & 20 & 1 & 21 & 1 & 28 & 1 & 33 & 1 \\
\hline IC 2581 & 7.14 & 0.42 & 11.94 & 0.021 & 31 & 0 & 35 & 0 & 39 & 0 & 42 & 1 \\
\hline \multicolumn{13}{|l|}{ GAC } \\
\hline NGC 7419 & 7.28 & 1.83 & 10.70 & 0.018 & 3 & 5 & 4 & 5 & 8 & 7 & 20 & 7 \\
\hline NGC 7235 & 7.07 & 0.93 & 12.25 & 0.017 & 19 & 2 & 25 & 2 & 36 & 2 & 60 & 2 \\
\hline NGC 2244 & 6.90 & 0.46 & 10.80 & 0.016 & 21 & 1 & 23 & 1 & 29 & 2 & 32 & 2 \\
\hline NGC 663 & 7.21 & 0.78 & 11.45 & 0.016 & 19 & 2 & 26 & 2 & 46 & 2 & 65 & 2 \\
\hline NGC 957 & 7.04 & 0.84 & 11.29 & 0.015 & 5 & 0 & 8 & 0 & 14 & 0 & 19 & 0 \\
\hline NGC 654 & 7.15 & 0.87 & 11.55 & 0.015 & 1 & 0 & 2 & 0 & 5 & 0 & 8 & 0 \\
\hline IC 1805 & 6.82 & 0.82 & 11.38 & 0.015 & 41 & 0 & 46 & 0 & 70 & 1 & 96 & 3 \\
\hline NGC 869 & 7.07 & 0.58 & 11.59 & 0.015 & 22 & 0 & 29 & 0 & 47 & 0 & 66 & 0 \\
\hline NGC 457 & 7.32 & 0.47 & 11.93 & 0.015 & 8 & 1 & 11 & 1 & 14 & 1 & 21 & 1 \\
\hline NGC 884 & 7.03 & 0.56 & 11.85 & 0.014 & 22 & 5 & 24 & 5 & 41 & 5 & 51 & 5 \\
\hline NGC 2439 & 7.25 & 0.41 & 12.93 & 0.013 & 7 & 1 & 8 & 1 & 14 & 1 & 22 & 1 \\
\hline \multicolumn{13}{|l|}{ LMC } \\
\hline NGC 2004 & 6.9 & 0.13 & 18.50 & 0.007 & 29 & 7 & 41 & 7 & 58 & 7 & 72 & 8 \\
\hline NGC 2100 & 7.2 & 0.13 & 18.50 & 0.007 & 19 & 11 & 20 & 12 & 33 & 13 & 39 & 13 \\
\hline NGC 1818 & 7.4 & 0.13 & 18.50 & 0.007 & 12 & 6 & 18 & 6 & 32 & 7 & 54 & 8 \\
\hline \multicolumn{13}{|l|}{$\mathrm{SMC}$} \\
\hline NGC 330 & 7.0 & 0.06 & 18.85 & 0.002 & 67 & 20 & 79 & 21 & 139 & 23 & 211 & 40 \\
\hline
\end{tabular}

Table 3. $B / R$ ratio for the galactic centre (GC), the galactic anticentre (GAC), SMC and LMC. $B$ in the column called "Spectroscopy" only includes B supergiants, while $R$ includes $\mathrm{K}$ and $\mathrm{M}$ supergiants. In the other columns, $B$ includes stars with $(B-V)_{0} \leq 0$ and $R$ stars with $(B-V)_{0} \geq 1.2$ (see text).

\begin{tabular}{|c|c|c|c|c|c|c|c|c|c|c|c|c|c|c|c|c|}
\hline & \multirow[b]{2}{*}{$Z$} & \multicolumn{3}{|c|}{ Spectroscopy } & \multicolumn{3}{|c|}{$M_{v \lim }=-3.25$} & \multicolumn{3}{|c|}{$M_{v \lim }=-3.0$} & \multicolumn{3}{|c|}{$M_{v \lim }=-2.5$} & \multicolumn{3}{|c|}{$M_{v \lim }=-2.0$} \\
\hline & & $B^{\dagger}$ & $R$ & $B^{\dagger} / R$ & $B$ & $R$ & $B / R$ & $B$ & $R$ & $B / R$ & $B$ & $R$ & $B / R$ & $B$ & $R$ & $B / R$ \\
\hline GC & 0.023 & 41 & 10 & 4.1 & 189 & 7 & 27.0 & 221 & 8 & 27.6 & 308 & 9 & 34.2 & 416 & 13 & 32 \\
\hline GAC & 0.016 & 29 & 23 & 1.3 & 168 & 17 & 9.9 & 206 & 17 & 12.1 & 324 & 21 & 15.4 & 460 & 23 & 20.0 \\
\hline LMC & 0.007 & - & - & - & 60 & 24 & 2.5 & 79 & 25 & 3.2 & 123 & 27 & 4.6 & 165 & 29 & 5.7 \\
\hline $\mathrm{SMC}$ & 0.002 & 9 & 15 & 0.6 & 67 & 20 & 3.4 & 79 & 21 & 3.8 & 139 & 23 & 6.0 & 211 & 40 & 5.3 \\
\hline
\end{tabular}

$\dagger$ Counting only B supergiants.

region. The increase of the $B / R$ ratio when the limit magnitude increases is simply due to the fact that more main sequence stars are included in $B$. The results are stable for the limit magnitudes $-3.25,-3.0$, and -2.5 , for which $B / R$ is approximately 2.5 times higher in the GC than in the GAC (in good agreement with Meylan \& Maeder 1982 and with the results from spectroscopic counts). For the limit magnitude of -2.0 , the value of $B / R$ for the GC seems relatively low, which likely reflects problems of completeness.

The $B / R$ ratios in the LMC (Table 3 ) confirm the decrease of $B / R$ when the metallicity decreases. However, the results for the SMC seem less clear. This is probably due to the fact that ratios for the SMC are based on one single cluster (NGC 330). This is obviously insufficient to obtain precise $B / R$ ratios and thus, in this case, results must be regarded with circumspection.
What are the effects of grouping clusters of different ages on the results? Let us recall that we selected clusters with log age between 6.8 and 7.5. This means that the $B / R$ ratio we obtain are for supergiants with initial masses between about 8 and $30 M_{\odot}$. Adopting narrower age intervals would give indication on the $B / R$ ratio in a smaller range of initial masses. One can wonder to which extent the decrease of the $B / R$ ratio with the metallicity would remain the same, if smaller age (mass) intervals were adopted. To check this point, we considered different age intervals (i.e. different intervals of initial masses for the supergiants). It is obvious that a finer age interval means less clusters selected and so a less reliable statistic. Therefore, we choose larger distance intervals when finer age intervals are considered. First, we calculate the $B / R$ ratio in the same three distance intervals considered above (6.5-8.0, 8.0-9.0 and $9.0-11.5 \mathrm{kpc}$ ) for two 


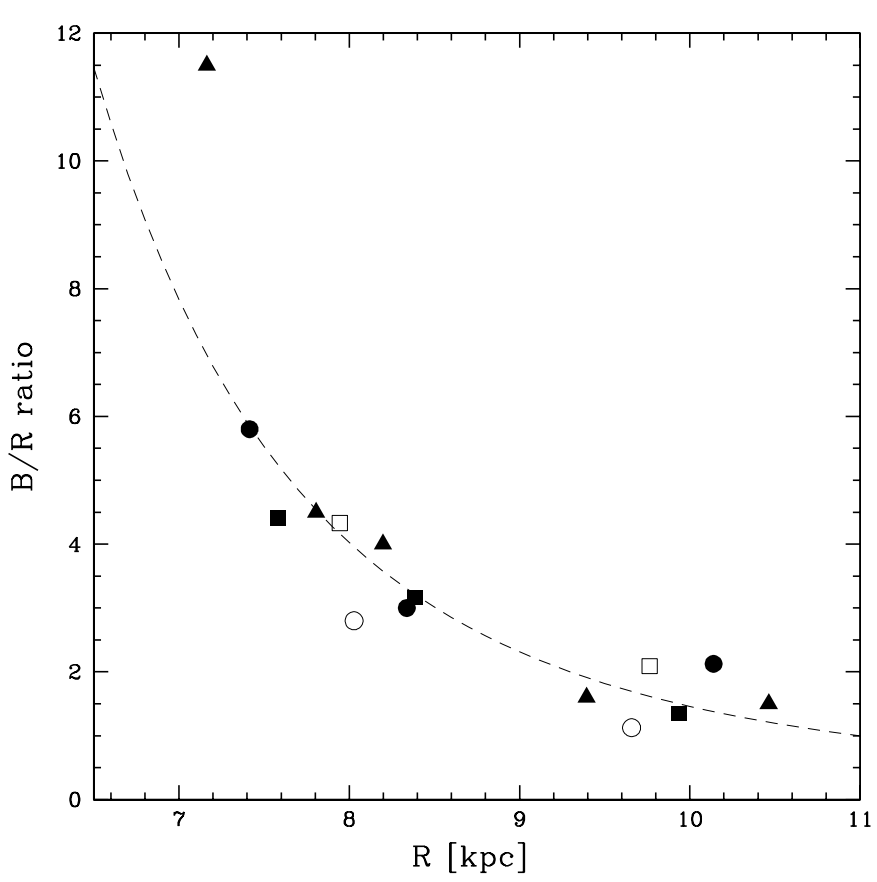

Fig. 2. $B / R$ ratio in the Galaxy for different age intervals, with distinction between blue and red supergiants based on spectroscopic measurements. B includes O, B and A supergiants. As in Fig. 1, triangles correspond to five different distance intervals in the Galaxy $(6.5-7.5,7.5-8.0,8.0-8.5,8.5-$ 10.0 and $10.0-11.5 \mathrm{kpc}$ ). Filled squares and circles correspond to three distance intervals in the Galaxy $(6.5-8.0,8.0-9.0$ and $9.0-11.5 \mathrm{kpc}$ ) and open squares and circles correspond to the two main distance intervals GAC and GC. Triangles include clusters with log age between 6.8 and 7.5 (i.e. initial masses of the supergiants between $\sim 8$ and $30 M_{\odot}$ ). Filled circles refer to clusters with log age between 6.8 and 7.2 (initial masses of the supergiants between $12-30 M_{\odot}$ ), while filled squares include clusters with log age between 7.0 and 7.4 (initial masses of the supergiants between 9-18 $\left.M_{\odot}\right)$. Open squares correspond to clusters with $\log$ age between 6.9 and 7.1 (initial masses of the supergiants between $14-23 M_{\odot}$ ) and open circles to clusters with $\log$ age between 7.1 and 7.3 (initial masses of the supergiants between $\left.10-15 M_{\odot}\right)$. The dashed curve corresponds to the same fit as in Fig. 1.

age intervals: $\log$ age between 6.8 and 7.2 (initial masses of the supergiants between $12-30 M_{\odot}$ ) and log age between 7.0 and 7.4 (initial masses of the supergiants between $\left.9-18 M_{\odot}\right)$. We also choose two finer age intervals, $\log$ age between 6.9 and 7.1 (initial masses of the supergiants between $14-23 M_{\odot}$ ) and log age between 7.1 and 7.3 (initial masses of the supergiants between 10-15 $M_{\odot}$ ), for which we calculate the $B / R$ ratio in the two main distance intervals GC and GAC. The results of these counts are shown in Fig. 2. We precise that the value of the galactocentric radius $R$ of each distance interval is obtained by averaging the galacocentric radii of the clusters found in the bin.

We observe that for different age intervals, the $B / R$ ratio always remains higher for larger metallicities. Figure 2 shows clearly that the variation of the $B / R$ ratio with the galactocentric radius remains more or less the same what- ever the interval of ages (masses) considered. Moreover, we can see that considering finer age intervals changes only slightly the values of the $B / R$ ratio. In that respect, the age interval between 6.8 and 7.5 appears to be a good compromise between the necessity to select a sufficiently high numbers of clusters to have a reliable statistics, and the necessity to somewhat restrain the domain of masses.

Finally, let us stress that when comparisons with stellar evolution models are made, the same definitions of the blue and the red supergiants used for obtaining the observed $B / R$ ratios has to be used to determine the theoretical $B / R$ ratios. In that respect, it is worthwhile to recall here that even if a star is classified as a blue supergiant, it may belong to the upper end of the Main Sequence.

\section{Conclusions}

The decrease of the $B / R$ ratio with the metallicity does not appear to be an artifact due for instance to incompleteness but instead a robust feature that stellar evolution models should be able to reproduce. The fact that the present day grids of stellar models are still unable to account for this feature demands some caution when evolutionary population synthesis models are used to interpret the integrated luminosity of high redshift galaxies.

Recent studies by Maeder \& Meynet (2001) show that the inclusion in the stellar models of the effects of rotation changes the $B / R$ ratios. Physically, this results from the mild mixing, which leads to more helium in the region of the $\mathrm{H}$-shell burning. The opacity is lower and the intermediate convective zone less important or absent. As convection implies a polytropic index $n=1.5$, which means a relative compactness of the internal convective regions, the absence of an intermediate convective zone is a necessary condition for stellar expansion to the supergiant stage. Interestingly, rotating models are able to account for the numerous red supergiants seen at low metallicity, a feature that standard models could not reproduce.

Acknowledgements. We would like to thank J.-C. Mermilliod for his help in using the Webda database.

\section{References}

Alibés, A., Labay, J., \& Canal, R. 2002, A\&A, submitted, [astro-ph/0107016]

Blaha, C., \& Humphreys, R. M. 1989, AJ, 98, 1598

Bomans, D. J., \& Grebel, E. K. 1994, Space Sci. Rev., 66, 61

Brocato, E., \& Castellani, V. 1992, Mem. S.A. Italy, 63, 121

Carney, B. W., Janes, K. A., \& Flower, P. J. 1985, AJ, 90, 1196

Cassatella, A., Barbero, J., Brocato, E., Castellani, V., \& Geyer, E. H. 1996, A\&A, 306, 125

Cayrel, R., Tarrab, I., \& Richtler, T. 1988, ESO Messenger, 54,29

Cervino, M., \& Mas-Hesse, J. M. 1994, A\&A, 284, 749

Cowley, A. P., Dawson, P., \& Hartwick, F. D. A. 1979, PASP, 91,628

Deng, L., Bressan, A., \& Chiosi, C. 1996, A\&A, 313, 159

Elias, J. H., Frogel, J. A., \& Humphreys, R. M. 1985, ApJS, 57,91 
Elson, R. A. W. 1991, ApJS, 76, 185

Freedman, W. L. 1985, AJ, 90, 2499

Grebel, E. K., \& Richtler, T. 1992, A\&A, 253, 359

Hagen, G. L., \& van den Bergh, S. 1974, ApJ, 189, L103

Hartwick, F. D. A 1970, ApJ, 7, 151

Humphreys, R. M. 1979a, ApJS, 39, 389

Humphreys, R. M. 1979b, ApJ, 231, 384

Humphreys, R. M., \& Davidson, K. 1979, ApJ, 232, 409

Humphreys, R. M., \& Davidson, K. 1984, Science, 223, 243

Humphreys, R. M., \& McElroy, D. B. 1984, ApJ, 284, 565

Humphreys, R. M., \& Sandage, A. R. 1980, ApJS, 44, 319

Ivanov, G. R. 1998, A\&A, 337, 39

Langer, N. 1991a, A\&A, 248, 531

Langer, N. 1991b, A\&A, 252, 669

Langer, N., \& Maeder, A. 1995, A\&A, 295, 685

Maeder, A., Grebel, E. K., \& Mermilliod, J.-C. 1999, A\&A, 346,459

Maeder, A., \& Meynet, G. 2001, A\&A, 373, 555

Massey, P. 2002, ApJS, 139, in press
McCarthy, J. K., Lennon, D. J., \& Venn, K. A. 1995, ApJ, 455, L135

Mermilliod, J.-C. 1995, in Information and On-Line data in Astronomy, ed. D. Egret, \& P. A. Albrecht (Kluwer), 127

Meylan, G., \& Maeder, A. 1982, A\&A, 108, 148

Meynet, G., Mermilliod, J.-C., \& Maeder, A. 1993, A\&AS, 98, 477

Origlia, L., Goldader, J. D., Leitherer, C., Schaerer, D., \& Oliva, E. 1999, ApJ, 514, 96

Pagel, B. E. J., Edmunds, M. G., Fosbury, R. A. E., \& Webster, B. L. 1978, MNRAS, 184, 569

Robertson, J. W. 1973, ApJ, 180, 425

Robertson, J. W. 1974, ApJ, 191, 67

Vallenari, A., Ortolani, S., \& Chiosi, C. 1994, A\&AS, 108, 571 van den Bergh, S. 1968, JRASC, 62, 69

van den Bergh, S. 1998, in The Galaxies of the Local Group (Cambridge University Press, Cambridge Astrophys. Ser.), 35,93

Walker, M. F. 1964, AJ, 69, 744 